\title{
Snippets in surgical pathology
}

\section{Journal of Clinical Pathology December 2009}

Rasty G, Hauspy J, Bandarchi B. Assessment of sentinel lymph node in cervical cancer: review of literature. J Clin Pathol 2009;62:1062-5.

A good overview of the role of sentinel node examination in uterine cervix cancer.

Butany J, Ahn E, Luk A. Drug-related cardiac pathology. J Clin Pathol 2009;62:1074-84.

Comprehensive and thorough overview of the cardiotoxic effects of a wide spectrum of drugs that are in routine use. Excellent resource if one encounters or is considering a drug-induced cardiac lesion.

Perez-Ordonez B. Hamartomas, papillomas and adenocarcinomas of the sinonasal tract and nasopharynx. J Clin Pathol 2009;62:1085-95.

Hamartomas, papillomas and sinonasal/ nasopharyngeal carcinomas are the topic of review in this 'My Approach' series. This author shares his experience with REAH, glandular (seromucinous) hamartona, exophytic/inverted/cylindrical cell papillomas and low-grade sinonasal/ intestinal type adenocarcinomas. Nicely written, easy to understand review of a potentially confusing area in head and neck pathology. (See also: Am J Surg Pathol 2009;33:401.)

Boyle DP, McCluggage WG. Combined actinomycotic and pseudoactinomycotic radiate granules in the female genital tract: description of a series of cases. J Clin Pathol 2009;62:1123-6.

This section features synopses of pertinent practical publications that appear in Pathology journals in the respective subspecialties. The summaries are mere guidelines and personal opinions of the two authors. The articles selected are diverse but occasionally reflect the authors' bias and are from the more widely read pathology journals. It is not intended to be an assiduous search of every publication in every Pathology journal, but more of a general indication of some of the monthly highlights through the eyes of the authors.

Hopefully, these snippets will provide the reader with enough to glean some facts and tips, as well as encourage them to read the entire article if necessary.
Actinomycosis of the gynaecological tract is well described in patients with intrauterine contraceptive devices. Pseudoactinomycotic radiate granules (PAMRAG) of the endometrium are also well recognised and should not be confused with organisms. This paper highlights the coexistence of both actinomycosis forming a central nidus (Gram, silver positive filamentous organisms) surrounded by PAMRAG with eosinophilic club-like structures (again not to confuse the latter with SplendoreHoeppli phenomenon). This study also documents these combined lesions in the cervix and vulva. PAMRAG comprise calcium, lipid, glycoprotein, copper, phosphate and iron.

\section{American Journal of Surgical Pathology December 2009}

Jagdis A, Rubin BP, Tubbs RR, et al. Prospective evaluation of TLE1 as a diagnostic immunohistochemical marker in synovial sarcoma. Am J Surg Pathol 2009;33:1743-51.

TLE1 encodes a transcriptional co-repressor and, using a commercially available antibody, was found to be overexpressed in $100 \%$ (with diffuse nuclear staining) of synovial sarcomas (a positive predictive value of $92 \%$ and a negative predictive value of $100 \%$ ).

Duncavage EJ, Le BM, Wang D, et al. Merkel cell polyomavirus: a specific marker for Merkel cell carcinoma in histologically similar tumors. $A m \mathrm{~J}$ Surg Pathol 2009;33:1771-7.

The Merkel cell polyoma virus is now an established oncogenic virus associated with Merkel cell carcinoma (occurring in about $50-80 \%$ of cases). This study shows that the virus is absent in other neuroendocrine carcinomas by PCR, establishing diagnostic specificity for Merkel cell carcinoma in this context.

Gerami P, Wass A, Mafee M, et al. Fluorescence in situ hybridization for distinguishing nevoid melanomas from mitotically active nevi. Am J Surg Pathol 2009;33:1783-8.

Fluorescence in situ hybridization for chromosome 6 and 11 aberrations distinguishes nevoid melanoma from mitotically active naevi.
Carmack SW, Vemupalli R, Spechler SJ, et al. Esophagitis dissecans superficialis ('sloughing esophagitis'): a clinicopathologic study of 12 cases. Am J Surg Pathol 2009;33:1789-94. Sloughing oesophagitis is seen occasionally and is also known as oesophagitis desiccans superficialis. This study documents the endoscopic findings (strips/ streaks of whitish 'pseudomembranous' material) and consistent histological findings of necrotic, sloughed/flaking superficial squamous epithelium. Rarely, bullous separation and rarely fungal elements are present. Possible aetiologies include drug medication and associated skin disorders.

Coco D. Hirsch M, Hornick JL. Smoothelin is a specific marker for smooth muscle neoplasms of the gastrointestinal tract. Am J Surg Pathol 2009;33:1795-801.

Smoothelin is a smooth-muscle-specific cytoskeletal protein exclusively found in differentiated smooth muscle cells. Other smooth muscle proteins (h-caldesmon, $\alpha$-smooth muscle actin, desmin, smooth muscle myosin) are expressed in proliferative (early) stages of smooth muscle development and occasionally in other cell types (striated muscle, myofibroblasts, myoepithelial cells, pericytes). Smoothelin has been shown to be expressed predominantly in visceral smooth muscle and to a lesser extent in vascular smooth muscle. This study explored smoothelin expression in a variety of spindled tumours occurring in the gastrointestinal tract. Diffuse cytoplasmic staining for smoothelin was seen in 100\% of leiomyomas; only $24 \%$ of leiomyosarcomas showed cytoplasmic positivity. None of the gastrointestinal stromal tumours showed cytoplasmic immunoreactivity. However, $41 \%$ of leiomyosarcomas and $22 \%$ of gastrointestinal stromal tumours (epithelioid/ mixed types) showed multifocal exclusively nuclear staining.

Zen Y, Onedera M, Inoue D, et al. Retroperitoneal fibrosis: a clinicopathologic study with respect to immunoglobulin G4. Am J Surg Pathol 2009;33:1833-9.

Zen Y, Inoue D, Kitao A, et al. IgG4-related lung and pleural disease: a clinicopathologic study of 21 cases. Am J Surg Pathol 2009;33:1886-93. IgG-4 related lymphoplasmacytic sclerosing disease is still in the news, being responsible for some retroperitoneal 
fibrosis (1833) and related lung/pleural disease (1886).

\section{Archives of Pathology and Laboratory Medicine \\ December 2009}

Al-Abbadi MA, Murthy R, Youngberg GA. Barrett oesophagus and the 'indefinite for dysplasia' category. Arch Pathol Lab Med 2009;133:1909.

Correspondence rarely features in these updates/snippets, but this letter/response to the editors addresses an often debated but poorly defined diagnosis of 'indefinite for dysplasia' in Barrett oesophagus and inflammatory bowel disease. These authors, contending that this is not an exact science, uses this diagnosis in three instances with a focus of columnar cell atypia: associated active inflammation, technical associated artefact, and deep crypt dysplasia with surface maturation.

Cornea V, Jaffer S, Bleiweiss IJ, et al. Adequate histologic sampling of breast magnetic resonance imaging-guided core needle biopsy. Arch Pathol Lab Med 2009;133:1961-4.

Most laboratories in developed countries are now faced with MRI-guided core needle biopsies of the breast (resulting in considerable major workload implications). This study demonstrates (with a cohort of 439 patients) that only one level is needed to arrive at an accurate diagnosis. This was demonstrated in about $95 \%$ of patients, with the remainder adding more information but not altering the diagnosis or patient outcome.

Weinreb I, Cunningham KS, Perez-Ordonez B, et al. CD10 is expressed in most epithelioid hemangioendoctheliomas: a potential diagnostic pitfall. Arch Pathol Lab Med 2009;133:1965-8. Another tumour that can be CD10 positive. Seven of nine primary epithelioid haemangioendotheliomas were positive.

\section{Histopathology \\ December 2009}

Wilkins BS, Clark DM. Making the most of bone marrow trephine biopsy. Histopathology 2009:55:631-40.

An excellent review of the bone marrow trephine biopsy that covers all practical, technical (including molecular techniques like immunohistochemistry, in situ hybridisation and PCR) aspects and, importantly, incorporates aspirate, peripheral blood and imaging results.

Lee S, Ogilvie RT, Dupre M, et al. Intravascular lymphocytosis in acute appendicitis: potential mimicry of chronic lymphocytic leukaemia. Histopathology 2009;55:660-4.

A long-noted diagnostic pitfall that many experienced surgical pathologists have encountered is the presence of intravascular lymphocytosis in acute appendicitis. This paper highlights this potential mimic of chronic lymphocytic leukaemia and attributes this phenomenon to surgical manipulation and the innate immunity of the patient.

\section{Diagnostic Histopathology December 2009}

This issue contains a mini-symposium on the pathology of the uterus and fallopian tube. There are good reviews on current concepts in tubal neoplastic pathology, the molecular genetics of endometrial carcinoma, and very practical piece on problematical areas in the reporting of endometrial carcinomas in hysterectomy specimens. Well worth reading.

\section{Human Pathology December 2009}

Meenakshi M, McCluggae WG. Myoepthelial neoplasms involving the vulva and vagina: report of four cases. Hum Pathol 2009:40:1747-53.

Myoepithelial tumours of the salivary gland, breast, skin and soft tissue are well described. This review of four cases covers the authors' experience in the vulva. As with all unusual tumours in unusual sites, awareness of morphology is critical to accurate diagnosis. Two tumours were composed entirely of ovoid or spindle-shaped cells, one was composed entirely of epithelioid cells, and in the other tumour there was a mixture of spindled and epithelioid cells. Small foci of ductal differentiation with squamous metaplasia were present in one case, and a minor stromal component, which varied from myxoid to hyalinised, was present in all cases. In all cases, the tumour cells were positive for epithelial markers (cytokeratins and/or epithelial membrane antigen) and the myoid markers, $\alpha$ smooth muscle actin and calponin. Desmin was positive in three cases. S100 and p63 were positive in one of the four neoplasms.

\section{Competing interests None.}

Provenance and peer review Not commissioned; not externally peer reviewed. 